Journal of Clinical Investigation

Vol. 42, No. 6, 1963

\title{
BIOCHEMICAL AND CHROMATOGRAPHIC STUDIES OF CERTAIN ACTIVITIES ASSOCIATED WITH HUMAN FIBRINOGEN PREPARATIONS *
}

\author{
By M. W. MOSESSON AND J. S. FINLAYSON
}

(From the Division of Biologics Standards, National Institutes of Health, Bethesda, Md.)

(Submitted for publication November 20, 1962; accepted February 7, 1963)

During the examination of human plasminogenfree fibrinogen, a stable heterogeneity of human fibrinogen was revealed by DEAE cellulose chromatography (1). Biophysical and biochemical studies have indicated that differences between the distinguishable types of fibrinogen are small and are best demonstrated electrophoretically or chromatographically.

In the present work, certain biological comparisons were made in a further attempt to characterize the different fibrinogen types. In addition to the properties considered to be intrinsic to fibrinogen (e.g., clotting time), we studied some biological activities that were found to be associated with fibrinogen and that probably represent nonfibrinogen contaminants.

We particularly examined the properties of a plasmin-trypsin inhibitor whose presence was first recognized during the course of these studies.

\section{MATERIALS AND METHODS}

Purified human fibrinogen was prepared from acid citrate dextrose plasma by the method of Blombäck and Blombäck (2) and subsequently reprecipitated from a solution containing lysine or $\epsilon-\mathrm{ACA}^{1}$ in order to remove plasminogen contamination (3). The final material was consistently $96 \%$ clottable or more and was stored in 0.3 $\mathrm{M} \mathrm{NaCl}$ at $-20^{\circ} \mathrm{C}$. Extensive dialysis was always performed to assure removal of free amino acid.

Glycerol-activated human plasmin, ${ }^{2} \quad 10.2 \pm 0.5$ casein $\mathrm{U}^{3}$ per $\mathrm{ml}$, was stored at $-20^{\circ} \mathrm{C}$ and diluted for use. Thrombin was a highly purified material ${ }^{4}$ of human

* Presented in part at the 9th Congress of the International Society of Hematology, September, 1962, Mexico City, Mexico.

${ }_{1} \epsilon$-ACA $=$ epsilon amino caproic acid.

2 Michigan State Laboratories, Lansing, Mich.

3 Assayed according to Sgouris, Inman, McCall, and Anderson's modification (4) of the method of Remmert and Cohen (5).

${ }^{4}$ Kindly supplied by Dr. D. L. Aronson, Laboratory of Blood and Blood Products, Division of Biologics Standards, National Institutes of Health, Bethesda, Md. origin containing no detectable plasmin or plasminogen activity at the concentrations employed. It was stored frozen at $-20^{\circ} \mathrm{C}$ in saline and diluted for use. Tryp$\sin ^{5}$ was stored at $-20^{\circ} \mathrm{C}$ in $0.001 \mathrm{~N} \mathrm{HCl}$ as a stock solution (10 $\mathrm{mg}$ per $\mathrm{ml})$ and was diluted as necessary for use.

Chromatography was performed on DEAE cellulose by the method previously described (6), using a deep concave gradient from starting buffer $\left(0.005 \mathrm{M} \mathrm{H}_{3} \mathrm{PO}_{4}\right.$ and $0.039 \mathrm{M}$ Tris, $\mathrm{pH} 8.6)$ to a final buffer $(0.5 \mathrm{M}$ $\mathrm{H}_{3} \mathrm{PO}_{4}$ and $0.5 \mathrm{M}$ Tris, $\left.\mathrm{pH} 4.1\right)$. The details of the heterogeneity revealed by chromatography have been presented elsewhere (1) and will be treated in this paper only as they pertain to subject matter.

Clottable protein was determined by a modification of the spectrophotometric method of Laki (7), described in detail elsewhere (1), which involves dilution of the sample with $0.05 \mathrm{M}$ phosphate buffer at $\mathrm{pH} 6.1$ to standard conditions of $\mathrm{pH}$ and ionic strength before coagulation with thrombin, incubation at room temperature for at least 1.5 hours, removal of the synerized clot with a Nichrome wire loop, and spectrophotometric measurement of the supernatant fluid at $280 \mathrm{~m} \mu$.

Thrombin clotting times were determined at $\mathrm{pH} 7.0$ in a medium $0.01 \mathrm{M}$ in phosphate and $0.135 \mathrm{M}$ in $\mathrm{NaCl}$. For each series of determinations, a thrombin concentration giving a clotting time of 13 to 17 seconds was chosen. Several concentrations of each sample of fibrinogen were then clotted with this concentration of thrombin. The time necessary for a visible fibrin film to form on a Nichrome wire loop was taken as the clotting time. Each determination was done in triplicate.

To study the sensitivities of various fibrinogen samples to plasmin or trypsin, a spectrophotometric method adaptable for measuring fibrinolysis or fibrinogenolysis was employed. Samples to be assayed were dialyzed against at least three changes of Tris-acetate- $\mathrm{NaCl}$ buffer at $\mathrm{pH}$ 8.0.6 After dialysis, the protein concentration was adjusted by dilution with the solution against which the final dialysis had been carried out. For fibrinolysis, $2.0 \mathrm{ml}$ of this buffered solution was pipetted into $15 \times$ $150-\mathrm{mm}$ test tubes and cooled to 0 to $5^{\circ} \mathrm{C}$ in an ice bath. To this was added $0.5 \mathrm{ml}$ of an appropriately

${ }^{5}$ Crystalline trypsin, Worthington Biochemical Corporation, Freehold, N. J.

6 $0.05 \mathrm{M}$ Tris in $0.275 \mathrm{M} \mathrm{NaCl}$ of which the $\mathrm{pH}$ was adjusted at room temperature to 8.0 with acetic acid; final ionic strength $=0.3$. 
diluted (in Tris-acetate- $\mathrm{NaCl}$ buffer) sample of plasmin or trypsin, followed after gentle mixing by $0.5 \mathrm{ml}$ of a Tris-buffered solution containing thrombin. The chilled mixture was then transferred to a $37^{\circ} \mathrm{C}$ water bath and allowed to incubate. The time required for all the additions and transfer to the water bath was only a few seconds. Visible clots formed less than 1 minute after transfer: solid clots, allowing complete inversion of the test tube without loss of contents, formed within 3 minutes. These conditions were usually achieved with a final concentration of thrombin in the range of 0.83 to $1.7 \mathrm{NIH} \mathrm{U}$ per $\mathrm{ml}$ ( 5 to $10 \mathrm{U}$ per $\mathrm{ml}$ in the thrombin solution used above). If solid clots did not form, as a result of relatively high plasmin or trypsin concentrations, higher concentrations of thrombin were used in repeated determinations. Once the thrombin concentration was sufficient to bring about solid clot formation, increasing it still further caused no change in the clot lysis rates determined as described below. This indicated that the lysis products appearing under the conditions of our experiments were from a clot formed in the presence of an excess of thrombin.

The solid clot that forms is, owing to the conditions of $\mathrm{pH}$ and ionic strength, translucent and nonsynerizing. As lysis progresses, the gel becomes opaque. (This phenomenon is more striking in the case of trypsin.) Reclarification of the clot then occurs before clot lysis, recorded as the time at which the gel breaks away from the sides of the vessel and the semisolid contents can be poured. In the present experiments, clot lysis usually occurred 10 minutes or more after transfer of the tubes to the water bath; however, precise measurement of the lysis time is difficult and is at best an approximation. Its measurement was actually unnecessary for our purposes, but, combined with the knowledge of substrate and enzyme concentrations, it served as a rough indicator of the intervals at which samples should be removed for spectrophotometric measurement (see below). Complete liquefaction, when no visible gel or suspended bubbles remained, occurred somewhat later; it was only after this event that the following quantitative measurements were performed.

After liquefaction, $0.5-\mathrm{ml}$ samples were removed at appropriate, timed intervals and transferred to $1.0 \mathrm{ml}$ of ice-cold ( 0 to $3^{\circ} \mathrm{C}$ ) $0.1 \mathrm{M}$ phosphate buffer at $\mathrm{pH} 6.1$. These were then diluted with $1.5 \mathrm{ml}$ of cold saline to a final volume of $3.0 \mathrm{ml}$ and allowed to stand for 15 to 30 minutes at this temperature before centrifugation for 20 minutes at $2,500 \mathrm{rpm}$ and $2^{\circ} \mathrm{C}$. The clear supernatant fluids were decanted, warmed to room temperature, and absorbance was read at $280 \mathrm{~m} \mu$. The phosphate buffer serves to lower the $\mathrm{pH}$ of the final solution to less than 6.3, which, in combination with the low temperature, effectively stops the reaction, i.e., there is no significant increase in absorbance of supernatant fluid when precipitated components remain in contact with solution longer than the usual time for separating precipitated components. Under these conditions, an opaque and synerizing gel reappears from the samples taken soon after lique- faction. Material reappearing from later samples becomes increasingly granular. Eventually, no granularity or cloudiness of any sort appears upon transfer to the phosphate solution. At any time during the removal of samples, the phenomena described permit easy separation of insoluble material from the supernatant fluid by centrifugation.

After about 20 to $30 \%$ of the total absorbance due to the substrate has appeared in the supernatant fluid, the absorbance of the supernatant fluid increases linearly with time. The lower the substrate concentration, the earlier the departure from linearity, but above a substrate concentration of $0.15 \%, 90 \%$ or more of the total absorbance appears before significant departure from linearity occurs. By holding the concentration of enzyme and substrate constant, one can obtain a linear plot of supernatant fluid absorbance against time, changes in the slope of which can be attributed to differences in substrate sensitivity or to the presence of various amounts of enzyme inhibitor, or both. The results are reproducible, and the variability in measured slope for any given set of conditions appears to be no more than about $10 \%$.

Fibrinogenolysis was determined by a similar technique. Fibrinogen samples were incubated with enzyme at $37^{\circ} \mathrm{C}$, and at appropriate time intervals, $0.5-\mathrm{ml}$ samples were withdrawn and mixed with $1.0 \mathrm{ml}$ of a solution containing at least $20 \mathrm{NIH} \mathrm{U}$ of thrombin in $0.1 \mathrm{M}$ phosphate buffer at $\mathrm{pH} 6.1$ at room temperature. At this $\mathrm{pH}$ and temperature, continued lysis does not contribute significantly to absorbance of the supernatant fluid, but clotting still occurs. The clots forming in samples taken relatively early in the incubation period are opaque and synerizing; those forming in progressively later samples are increasingly strand-like. After standing for about 30 minutes in the phosphate buffer, the samples were centrifuged and the supernatant fluid removed by decantation. The absorbance of the clear supernatant fluid was then measured at $280 \mathrm{~m} \mu$. Loss of clottability, i.e., increase in absorbance of supernatant fluid, was linear with time until about $10 \%$ of the initial clottable protein remained.

Fibrinase ${ }^{7}$ activity was determined by the method of Loewy, Dunathan, Kriel, and Wolfinger (8). Rough quantitation was achieved by incubating the samples for different periods with or without cysteine, which is known to increase the sensitivity. Any clot remaining soluble in $2 \%$ acetic acid after incubation in the presence of cysteine for 18 hours was considered to be fibrinase free. Fibrinase-free fibrinogen was obtained by chromatographing fibrinogen (see below). "Crude" fibrinase, free of fibrinogen, was prepared by the method outlined by Loewy and associates (8) and was carried as far as the chromatographic step (Fraction 5).

${ }^{7}$ The development of the nomenclature of this substance has been outlined by Loewy and associates (8). In this paper, we have used the names "fibrinase" and "fibrin-stabilizing factor" (FSF) interchangeably. 


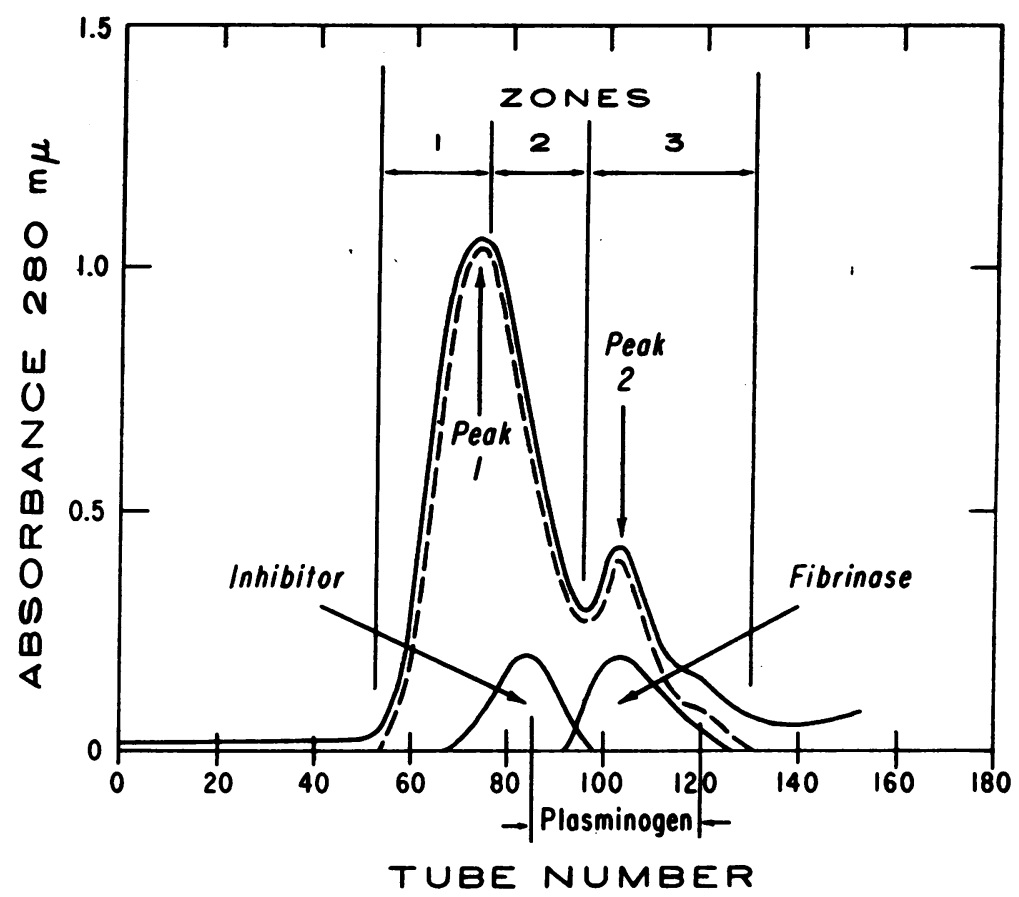

Fig. 1. Distribution of protein and biological activities in a fibrinoGEN CHROMATOGRAM. Material chromatographed was plasminogen-free human fibrinogen that was $97.8 \%$ clottable. Distribution of fibrinase activity was derived from data such as that in Table $I$. Distribution of inhibitor activity was obtained from measurements in a plasmin-fibrin system. Both fibrinase and inhibitor are represented in arbitrary units. The position in which plasminogen is eluted when fibrinogen samples contaminated with this proenzyme are chromatographed is included for comparison. Clottable protein is indicated by the dashed line. The absorbance scale on the ordinate pertains only to the chromatographic distribution of fibrinogen. Each tube contained $12 \mathrm{ml}$ of eluate.

\section{EXPERIMENTAL PROCEDURES AND RESULTS}

The elution pattern of highly purified fibrinogen always included two major peaks of clottable protein and a small third shoulder or peak (Figure 1). Peak 1 fibrinogen comprised $85 \pm 2 \%$ of the total; peak 2 fibrinogen amounted to $15 \pm$ $2 \%$. Peak 1 was, on the average, $98 \%$ clottable or more for any preparation more than 95\% clottable before chromatography. Peak 2 was usually $85 \%$ clottable or more, but this depended to a greater extent on the preparation chromatographed. The only significant difference found between these two types of fibrinogen was in the electrophoretic mobilities, which were consistent with the anionic binding capacities of the different types revealed by chromatography. That is, whereas both types migrated more slowly than $\beta$-globulin during paper electrophoresis at $\mathrm{pH} 8.6$, peak 2 migrated more rapidly toward the anode than did peak 1 . The mobility of peak 1 determined by moving-boundary electrophoresis at $\mathrm{pH}$ 8.6 is the same as that of unchromatographed fibrinogen, which consists primarily of peak 1 fibrinogen. Unchromatographed fibrinogen, however, consistent with its content of peak 2 fibrinogen, appears as an asymmetrical peak skewed toward the anode (1). No explanation is as yet available for the higher negative charge at $\mathrm{pH} 8.6$ of peak 2 fibrinogen.

For comparative purposes, column eluate was divided into three zones $(1,2$, and 3 in Figure 1). pooled, precipitated at $\mathrm{pH} 6$ to 7 with $10 \%$ ethanol at 0 to $-2^{\circ} \mathrm{C}$, redissolved in $0.3 \mathrm{M} \mathrm{NaCl}$. and dialyzed against appropriate solutions. Zone 
TABLE I

The fibrinase content of a column eluate and of various fibrinogen fractions*

\begin{tabular}{|c|c|c|c|c|c|c|c|c|c|c|c|c|c|c|c|c|c|c|}
\hline \multirow[b]{3}{*}{ Incubation } & \multirow{2}{*}{\multicolumn{16}{|c|}{ Fraction or tube number }} & \multicolumn{2}{|c|}{ Fibrinogen } \\
\hline & & & & & & & & & & & & & & & & & \multirow{2}{*}{$\begin{array}{c}\text { Fibrinase- } \\
\text { free } \\
\text { (peak 1) }\end{array}$} & \multirow{2}{*}{$\begin{array}{l}\text { Unchro- } \\
\text { mato- } \\
\text { graphed }\end{array}$} \\
\hline & 55 & 65 & 75 & 85 & 90 & 93 & 95 & 98 & 100 & 105 & 110 & 115 & 120 & 125 & 130 & 140 & & \\
\hline \multicolumn{19}{|c|}{ Without cysteine } \\
\hline $\begin{array}{l}1 \text { hour } \\
6 \text { hours }\end{array}$ & $\begin{array}{l}0 \\
0\end{array}$ & $\begin{array}{l}0 \\
0\end{array}$ & $\begin{array}{l}\mathbf{0} \\
0\end{array}$ & $\begin{array}{l}0 \\
0\end{array}$ & $\begin{array}{l}0 \\
0\end{array}$ & $\begin{array}{l}0 \\
0\end{array}$ & $\begin{array}{l}0 \\
0\end{array}$ & $\begin{array}{l}0 \\
+\end{array}$ & $\begin{array}{l}+ \\
+\end{array}$ & $\stackrel{+}{+}$ & $\begin{array}{l}0 \\
+\end{array}$ & $\begin{array}{l}\mathbf{0} \\
\mathbf{0}\end{array}$ & $\begin{array}{l}0 \\
0\end{array}$ & $\begin{array}{l}0 \\
0\end{array}$ & $\begin{array}{l}0 \\
0\end{array}$ & $\begin{array}{l}0 \\
0\end{array}$ & $\begin{array}{l}\mathbf{0} \\
\mathbf{0}\end{array}$ & $\begin{array}{l}+ \\
+\end{array}$ \\
\hline \multicolumn{19}{|l|}{ With cysteine } \\
\hline $\begin{array}{l}6 \text { hours } \\
18 \text { hours }\end{array}$ & $\begin{array}{l}0 \\
0\end{array}$ & $\begin{array}{l}0 \\
0\end{array}$ & $\begin{array}{l}0 \\
0\end{array}$ & $\begin{array}{l}0 \\
0\end{array}$ & $\begin{array}{l}0 \\
0\end{array}$ & $\begin{array}{l}0 \\
+\end{array}$ & + & + & + & + & $\begin{array}{l}+ \\
+\end{array}$ & $\begin{array}{l}+ \\
+\end{array}$ & $\begin{array}{l}+ \\
+\end{array}$ & $\begin{array}{l}0 \\
+\end{array}$ & $\begin{array}{l}0 \\
0\end{array}$ & $\begin{array}{l}0 \\
0\end{array}$ & $\begin{array}{l}\mathbf{0} \\
0\end{array}$ & $\begin{array}{l}+ \\
+\end{array}$ \\
\hline
\end{tabular}

${ }^{*}$ Sensitivity of the assay increases from top to bottom of table. $0=$ clot dissolves within 24 hours in $2 \%$ acetic acid medium, and $+=$ insoluble clot after 24 hours in $2 \%$ acetic acid medium.

1 represented exclusively peak 1 material; zone 2 , predominantly peak 1 material; and zone 3 , predominantly, although not entirely, peak 2 fibrinogen.

Clotting times were compared for various chromatographic fractions from several columns and for several unchromatographed fibrinogen preparations. There were no significant differences in the thrombin clotting times.

Fibrinogen preparations were assayed for their content of fibrinase. Plasminogen-free fibrinogen was found to contain about the same amount of clot-stabilizing activity as untreated fibrinogen. Examination of the chromatographic zones revealed that even after 18 hours of incubation in the presence of cysteine, there was no detectable fibrinase activity in zone 1 , whereas appreciable amounts were present in zone 3. Peak 1 was considered to be free of fibrinase, and served as a convenient and ample source of fibrinogen for the purposes of fibrinase assay. More precise location of fibrinase activity was then achieved by screening a column for fibrinase activity by using peak 1 prepared from a previous column (Table I, Figure 1). Fibrinase activity was found to be greatest in the region of tubes 100 to 105 . There was no detectable clot-stabilizing activity until at least tube 90 . This finding corroborated the previous observations of the fibrinase contents of zones 1 to 3 . Moreover, peak 1 fibrinogen from many chromatographic runs of several different batches of fibrinogen has never exhibited detectable fibrin-stabilizing activity.

Because of our interest in developing a suitable fibrinogen preparation for use in fibrinolytic assay procedures, we compared fibrinogen samples for sensitivity to plasmin and to trypsin. Plasmin was used in both fibrinolytic and fibrinogenolytic analyses, and trypsin, primarily in fibrinogenolytic. $^{8}$ All samples were dialyzed extensively to assure removal of lysine or $\epsilon$-ACA. Furthermore, when comparative studies were performed, all samples were dialyzed in the same vessel.

It was first noted that the material from the earlier part of the chromatogram (tubes 55 to 80 ) lysed at a greater rate than unchromatographed fibrinogen. Moreover, recombination of the earlier part of peak 1 with peak 2 in any proportions always yielded a mixture that lysed more rapidly than unchromatographed fibrinogen. Therefore, chromatographic zones 1 to 3 were prepared and their lysis rates compared by the methods described above (i.e., with plasmin for fibrinolysis and fibrinogenolysis, trypsin for fibrinogenolysis).

${ }^{8}$ We have confirmed the observations of others $(9,10)$ that trypsin digests fibrinogen far more rapidly than it does a fibrin clot. We believe that this phenomenon is due to the fact that trypsin is not incorporated in the gel phase of a clot as plasmin is and therefore may be excluded from intimate association with its substrate. Although it is beyond the scope of this paper to present detailed data in support of this contention, the supposition is based essentially upon the fact that a major portion of trypsin added to fibrinogen before coagulation with thrombin can be recovered immediately in the supernatant fluid from the synerized clot, and little more of the enzyme can be recovered as clot lysis progresses (11). Plasmin, on the other hand, appears in the supernatant fluid in relation to the degree of clot lysis. Whatever the reason, however, we felt that the reaction in the trypsin-fibrin system was too complex for us to attempt demonstration of inhibitory activity. 


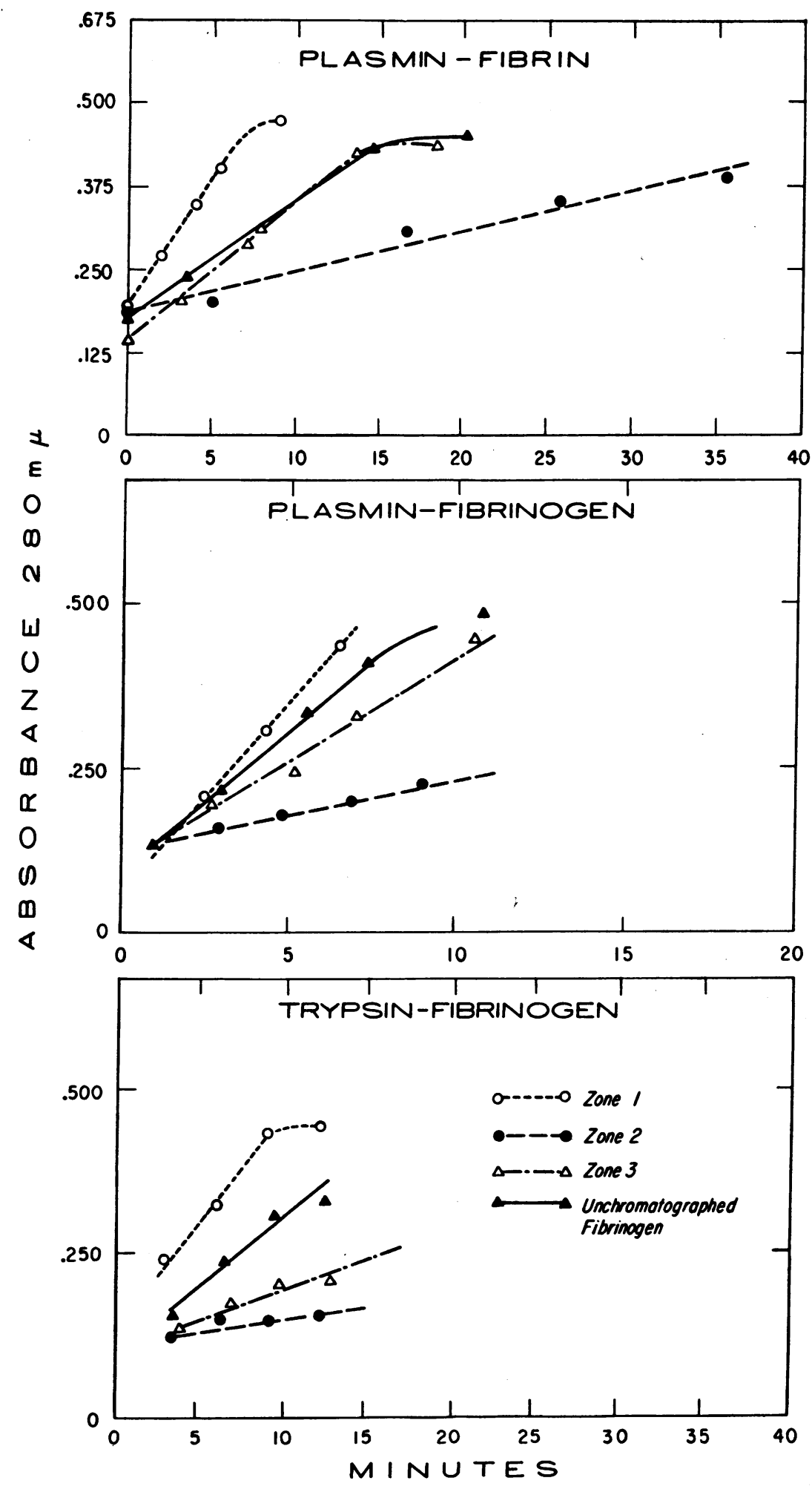

Fig. 2. HYDROLYSIS OF VARIOUS FIBRINOGEN FRACTIONS AT CONSTANT SUBSTRATE AND ENZYME CONCENTRATIONS. The final concentration of fibrinogen in the incubation mixture was, in every case, about $0.17 \%$. Final concentration of plasmin in the fibrinogen or fibrin systems was 0.085 casein $U$ per $\mathrm{ml}$, whereas the final concentration of trypsin in the fibrinogen system was $1.6 \mu \mathrm{g}$ per $\mathrm{ml}$. The experiments in a given enzyme-substrate system were conducted simultaneously.

The designations of the substrates $(O, \bullet, \Delta$, and $\mathbf{\Delta})$ are indicated in the legend of the bottom graph and are the same throughout the figure. 


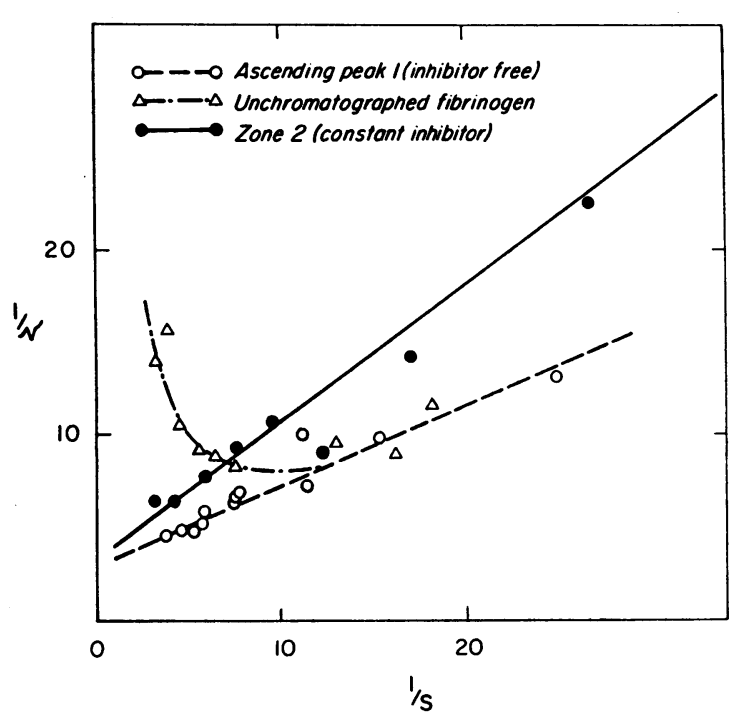

Fig. 3. Double-Reciprocal plot (Lineweaver-Burk) in THE FIBRIN-PLASMIN SYSTEM. Plasmin concentration in these studies was constant at 0.0485 casein $\mathrm{U}$ per $\mathrm{ml}$. $1 /$ reaction velocity $(1 / v)$ is expressed as change in absorbance of supernatant fluid at $280 \mathrm{~m} \mu$ per $10 \mathrm{~min}$ utes $^{-1}\left(\Delta A_{280} / 10 \mathrm{~min}^{-1}\right)$. 1/substrate concentration (1/S) is expressed as the percentage of fibrinogen in the assay mixture $^{-1}\left(\%^{-1}\right)$.

All methods yielded qualitatively similar results. The fibrinogen from zone 2 lysed at a rate that was even slower than that of unchromatographed fibrinogen. Zone 1 fibrinogen always lysed at the greatest rate, whereas zone 3 fibrinogen lysed at a rate between those of zones 1 and 2 (Figure 2). Examination of chromatographic fractions from several different batches of fibrinogen yielded qualitatively similar results, and the lysis rate of a given zone 2 preparation was consistent with that of the corresponding unchromatographed fibrinogen. In a series of mixtures prepared from the three zones, the lysis rates correlated with the amount of zone 2 in the mixture, rather than with the amount of peak 1 or peak 2 fibrinogen calculated to be present. Thus the observed differences appeared to be due to the presence of a plasmintrypsin inhibitor previously unsuspected in fibrinogen of this high degree of purity.

Successive chromatographic fractions from a single experiment were then assayed to locate the region of maximal inhibition. Fractions were dialyzed against saline before assay. The ascending limb (up to about tube 70) of peak 1 was pooled and precipitated. This fraction will be re- ferred to as ascending peak 1. It was assumed to be inhibitor free (an assumption supported by kinetic studies described below). Samples of column fractions were added to this "inhibitorfree" fibrinogen, and the rates of fibrinolysis of resulting mixtures were determined. Measurable inhibition of lysis began at about tube 70 and reached a maximum in the descending limb of peak 1 (Figure 1). This accounted for our earlier observations of greatest activity in the zone between the two peaks.

The nature of the inhibition has been partially investigated. The activity could not be found in the supernatant fluid after clotting. It did not appear to be soluble in 5\% trichloroacetic acid; however, possible losses of activity during the procedure could not be assessed. It has not been found in the fibrinase-rich fraction of fibrinogen that remains soluble after heating to $56^{\circ} \mathrm{C}$ for 3 minutes. The inhibitor has been further differentiated from fibrinase by addition of preparations of fibrinogen-free fibrinase to fibrinasefree fibrinogen in amounts sufficient to detect fibrin-stabilizing activity; under these conditions no inhibitory properties could be demonstrated. Moreover, the chromatographic properties of the two activities are distinct (Figure 1).

Additional kinetic studies have been carried out with the plasmin-fibrin system to test the assumption that the early portion of peak 1 contains no detectable inhibitory activity and to ascertain the type of inhibition involved. Plasmin concentration was held constant and substrate concentration was varied. Data was plotted in the double-reciprocal manner of Lineweaver and Burk (12). Plotting the data in the manner suggested by Eadie (13) and Hofstee (14) gave similar information. Within the range of concentrations studied $(0.087$ to $0.26 \%)$, fibrinogen from ascending peak 1 yielded a straight line (Figure 3).

The experiments shown in Figure 3 were performed on material from a single batch of fibrinogen. However, the lysis rates of ascending peak 1 samples from several different fibrinogen preparations were independent of the lysis rates (and hence, presumably, of the inhibitor contents) of the corresponding unchromatographed preparations. When compared in the double-reciprocal manner, the various ascending peak 1 preparations 
yielded straight lines, the slopes of which did not appear to differ from one another at a given enzyme concentration.

For determining the type of inhibition, zone 2 (containing relatively high inhibitory activity) was added to a series of solutions of "inhibitorfree" fibrinogen (ascending peak 1) to yield mixtures containing constant amounts of zone 2 fibrinogen $(0.037 \%)$, but varying amounts of total fibrinogen. Lysis rates were then determined and the data plotted. A line drawn through the points would appear to intercept the plot of ascending peak 1 at the ordinate, thus indicating that with respect to plasmin acting on a fibrin substrate, the inhibitor is competitive (Figure 3 ).

The unchromatographed fibrinogen was also studied under the same conditions. In unchromatographed fibrinogen, there is a constant inhibitor: substrate ratio, so as the preparation is diluted to adjust substrate concentration, a corresponding alteration in inhibitor concentration occurs. At low substrate concentrations, where the amount of inhibitor was small with respect to enzyme concentration, the observed lysis rate approached that of "inhibitor-free" fibrinogen at a corresponding concentration (Fig. 3). At higher substrate concentrations, the inhibitor concentration was also higher, and its activity was manifested by decreasing lysis rates. The curve obtained for unchromatographed fibrinogen (Figure 3) was nonlinear. The points on it may be thought of as points from a family of straight lines corresponding to different inhibitor concentrations. Moreover, it could be estimated from the intercepts of their curves that zone 2 contained about 3.8 times as much inhibitor per milligram fibrinogen as did unchromatographed fibrinogen. For this preparation of unchromatographed fibrinogen, the inhibitory activity was insignificant below a substrate concentration of $0.09 \%$; however, the amount of inhibitor in unchromatographed fibrinogen varies from preparation to preparation, so that this value is not constant. Finally, the linearity of the data obtained from fibrinogen from ascending peak 1, especially when compared with unchromatographed fibrinogen, supports the assumption that within the range studied, there are no significant amounts of inhibitor present in this fraction.

Because of the presence of the inhibitory ac- tivity, it has been difficult to determine directly whether there actually are any differences in the sensitivities of peak 1 and peak 2 fibrinogen to plasmin or trypsin, but kinetic data imply that differences, if any, are subtle. Immunoelectrophoretic studies of the products of plasmin or trypsin digestion have failed to reveal any differences between the different fibrinogens (15).

\section{DISCUSSION}

The fibrinogenolytic assay employed in these studies involves thrombin only after the allotted digestion period with trypsin or plasmin. The addition of thrombin before significant digestion of fibrinogen has occurred is the basis for what we have termed the fibrinolytic assay, for the reasons summarized below.

Under the conditions of this assay, thrombin is in excess, since when solid clot formation does occur with a given concentration of thrombin. higher concentrations of thrombin do not induce any change in the rate of appearance of soluble digestion product. The appearance of soluble product in the fibrinolytic assay is slower than in the fibrinogenolytic system under equivalent plasmin and substrate concentrations (Figure 2). Moreover, at the time that liquefaction, or for that matter, clot lysis, occurs in the fibrinolytic assay, no clottable protein is detectable in the fibrinogenolytic assay. (This difference is much greater in the case of trypsin digestion, and clot lysis occurs long after the time when no clottable protein exists in the fibrinogenolytic system. ${ }^{8}$ ) Since it is only after complete liquefaction that samples are taken for measurement, no fibrinogen would be expected to remain at the time of assay. Furthermore, the insoluble material obtained in phosphate buffer in the fibrinolytic system can be redissolved in the original volume of the $\mathrm{pH} 8$ Tris buffer, containing $0.1 \mathrm{M} \epsilon$-ACA to inhibit further lysis, and reprecipitated by cold phosphate buffer. On the other hand, the clot obtained in the fibrinogenolytic assay remains insoluble in the original volume of $\mathrm{pH} 8 \mathrm{Tris}$ buffer containing $0.1 \mathrm{M} \epsilon$-ACA. Finally, immunoelectrophoretic studies (15) of fibrin and fibrinogen digestion products with rabbit antihuman fibrinogen and using soy bean inhibitor to inhibit plasmin indicate that, whereas the final products of fibrinolysis, i.e., those formed 
after 24 hours of incubation, are indistinguishable from those of fibrinogenolysis, the products present during the periods of assay in the two systems are distinguishable.

Little is known of the nature of the product reappearing in the phosphate buffer in the fibrinolytic assay. It is probably a phenomenon similar to that described by Derechin and Szuchet (16), who were able to reinduce gel formation of fibrin lysed by plasmin by adding various organic and inorganic reagents, as well as by reducing the ionic strength by dilution with water. That is, it is apparently the reformation of gel or insoluble material by alteration of conditions to those less favorable for the solubility of a partially hydrolyzed fibrin product. The fact that under the present conditions soluble product appears linearly with time has encouraged us to develop this assay as a means of enzyme quantitation; it would appear to be as valid a measure of fibrinolysis as any clot lysis assay.

The only apparent effect of lysine or $\epsilon$-ACA treatment on fibrinogen has been the elimination of plasminogen as a contaminant. No biophysical changes have been noted (3), nor have there been any changes in the biological properties other than those attributable to plasminogen. One of the biological properties associated with fibrinogen and, until recently, inseparable from it has been fibrinase. Loewy and associates (8) used repeated fractional precipitation, resulting in very low yields, in order to obtain fibrinogen sufficiently low in fibrinase to serve as a substrate in its measurement. In a chromatographic experiment on fibrinogen (species not specified), Lorand observed that the first fractions eluted, comprising only a minor portion of the total clottable protein, were apparently free of fibrin-stabilizing factor, whereas later fractions were heavily contaminated (17). Although it represents an incidental finding in our chromatographic and biological studies, the observation that the fibrinogen in peak 1 is free of fibrinase indicates that this chromatographic procedure is a more efficient preparative method than the ammonium sulfate fractionation.

Shulman (18) reported the isolation of a plasmin and trypsin inhibitor from human plasma and urine; this material did not inhibit thrombin, and it was soluble in trichloroacetic acid. The only plasma fraction from which he was able to prepare the material was the euglobulin fraction, and in view of its small molecular weight and solubility properties, he proposed that the inhibitor was bound to the proteins contained in this fraction. With the finding of the inhibitory activity in fibrinogen, which behaves like an euglobulin, a possible relationship between these two inhibitors was suggested. Several attempts at preparing a fibrinogen-free inhibitor from the cold-insoluble fraction of Cohn Fraction I, purified fibrinogen, or chromatographic zone 2 according to Shulman's method (18) have so far been unsuccessful. but investigations are continuing. In 1952 (19), Shulman suggested that a plasmin inhibitor might be present in bovine fibrin. The inhibitory activity was not apparent with trypsin or chymotrypsin. Astrup and Müllertz (20), using a bovine fibrin substrate of low purity, described an antitryptic activity which they felt was more prominent than the antiplasmin activity. What relation, if any, the bovine inhibitor may have to the one present in human material is obscure.

The possibility that the observed inhibition might represent the effect of residual lysine has been ruled out on several grounds. No differences in lysis rates could be found by comparing fibrinogen from which an added amount of lysine had been dialyzed with fibrinogen to which no lysine had been added. No binding of lysine to fibrinogen could be demonstrated by equilibrium dialysis (3). Bergström and Wallén (21), using the sensitive technique of $\mathrm{N}$-terminal amino acid analysis, could find no lysine in bovine fibrinogen after dialysis. Using a similar technique on human fibrinogen, we have not found any detectable lysine.

The significance of a plasmin inhibitor associated with fibrinogen remains to be determined with regard to clot lysis in vivo. At the moment, its potential importance lies in effects it may have on the application of fibrinolytic or fibrinogenolytic assays to the measurement of proteolytic enzymes (primarily plasmin). Objections have been raised to most if not all of the substrates proposed for plasmin assay (22). Until recently, the variable plasminogen contamination of fibrinogen posed difficulties of standardization that led to the adoption of a casein substrate (23). However, "casein" itself can be separated into several types of 
caseins with different sensitivities to plasmin (24, 25). The need for the careful choice of wellcharacterized substrates for use in assay procedures is obvious.

\section{SUM M ARY}

Studies were made of biological activities associated with the types of human fibrinogen distinguishable by their chromatographic behavior on DEAE cellulose. No significant differences in thrombin clotting times were detected. A previously unsuspected inhibitor of plasmin and trypsin, present in all unchromatographed fibrinogen preparations examined, was revealed by comparison of the sensitivities of various chromatographic fractions of fibrinogen to digestion by plasmin and trypsin. Kinetic data indicated that with respect to plasmin acting on a fibrin substrate the inhibitor was competitive. The ascending portion of the first major chromatographic peak was found to be free of inhibitor activity within the range of substrate concentrations studied. The inhibitor was not identical with fibrinase. Chromatography was an efficient method for preparing fibrinasefree fibrinogen.

\section{REFERENCES}

1. Finlayson, J. S., and M. W. Mosesson. Heterogeneity of human fibrinogen. Biochemistry 1963, 2, 42.

2. Blombäck, B., and M. Blombäck. Purification of human and bovine fibrinogen. Arkiv Kemi 1956, $10,415$.

3. Mosesson, M. W. The preparation of human fibrinogen free of plasminogen. Biochim. biophys. Acta (Amst.) 1962, 57, 204.

4. Sgouris, J. T., J. K. Inman, K. B. McCall, and J. D. Anderson. The evaluation of "fibrinolytic" preparations. Vox Sang. (Basel) 1962, 6, 53.

5. Remmert, L. F., and P. P. Cohen. Partial purification and properties of a proteolytic enzyme of human serum. J. biol. Chem. 1949, 181, 431.

6. Finlayson, J. S., R. T. Suchinsky, and A. L. Dayton. Effects of long-term storage on human serum albumin. I. Chromatographic and ultracentrifugal aspects. J. clin. Invest. 1960, 39, 1837.

7. Laki, K. The polymerization of proteins: the action of thrombin on fibrinogen. Arch. Biochem. 1951, $32,317$.
8. Loewy, A. G., K. Dunathan, R. Kriel, and H. L. Wolfinger, Jr. Fibrinase I. Purification of substrate and enzyme. J. biol. Chem. 1961, 236, 2625.

9. Lewis, J. H., and J. H. Ferguson. Studies on a proteolytic enzyme system of the blood. V. Activation of profibrinolysin by trypsin. Amer. J. Physiol. 1952, 170, 636.

10. Cliffton, E. F., and D. Cannamela. Fibrinolytic and proteolytic activity of a human plasminogen prepared from fraction III of human plasma. $J$. appl. Physiol. 1953, 6, 42.

11. Mosesson, M. W. Unpublished data.

12. Lineweaver, H., and D. Burk. The determination of enzyme dissociation constants. J. Amer. chem. Soc. 1934, 56, 658.

13. Eadie, G. S. On the evaluation of the constants $V_{m}$ and $K_{\mu}$ in enzyme reactions. Science 1952, 116, 688.

14. Hofstee, B. H. J. On the evaluation of the constants $V_{m}$ and $K_{m}$ in enzyme reactions. Science 1952, 116, 329.

15. Yokoyama, M., and M. Mosesson. Unpublished results.

16. Derechin, M., and S. Szuchet. Gelation phenomena in degraded fibrinogen and fibrin. Arch. Biochem. 1960, 87, 100.

17. Lorand, L. Properties and significance of the fibrin stabilizing factor (FSF). Thrombos. Diathes. haemorrh. (Stuttg.) 1962, 7, suppl. 1, 238.

18. Shulman, N. R. A proteolytic inhibitor with anticoagulant activity separated from human urine and plasma. J. biol. Chem. 1955, 213, 655.

19. Shulman, N. R. Studies on the inhibition of proteolytic enzymes by serum. I. The mechanism of the inhibition of trypsin, plasmin, and chymotrypsin by serum using fibrin tagged with $\mathrm{I}^{131}$ as a substrate. J. exp. Med. 1952, 95, 571.

20. Astrup, T., and S. Müllertz. The fibrin plate method for estimating fibrinolytic activity. Arch. Biochem. 1952, 40, 346.

21. Bergström, K., and P. Wallén. Removal of contaminating plasminogen from purified bovine fibrinogen. Arkiv Kemi 1961, 17, 503.

22. Sherry, S., A. P. Fletcher, and N. Alkjaersig. Fibrinolysis and fibrinolytic activity in man. Physiol. Rev. 1959, 39, 343.

23. Advisory Committee on Coagulation Products, Protein Foundation, Boston. Investigations of fibrinolysis and labeling changes in fibrinolysin products. J. Amer. med. Ass. 1962, 180, 536.

24. Christensen, L. R. The action of proteolytic enzymes on casein proteins. Arch. Biochem. 1954, $53,128$.

25. Derechin, M. Hydrolysis of some casein fractions with plasma. Biochem J. 1962, 82, 42. 